\title{
A T8.5 BROWN DWARF MEMBER OF THE $\xi$ URSAE MAJORIS SYSTEM
}

\author{
Edward L. Wright ${ }^{1}$, M. F. Skrutskie ${ }^{2}$, J. Davy Kirkpatrick ${ }^{3}$, Christopher R. Gelino $^{3}$, Roger L. Griffith $^{3}$, \\ Kenneth A. Marsh ${ }^{4}$, Tom Jarrett ${ }^{3}$, M. J. Nelson ${ }^{2}$, H. J. Borish ${ }^{2}$, Gregory Mace ${ }^{1}$, Amanda K. Mainzer ${ }^{5}$, \\ Peter R. Eisenhardt ${ }^{5}$, Ian S. McLean ${ }^{1}$, John J. Tobin ${ }^{6}$, and Michael C. Cushing ${ }^{7}$ \\ ${ }^{1}$ UCLA Astronomy, P.O. Box 951547, Los Angeles, CA 90095-1547, USA; wright@ astro.ucla.edu \\ ${ }^{2}$ Department of Astronomy, University of Virginia, Charlottesville, VA 22904, USA \\ ${ }^{3}$ Infrared Processing and Analysis Center, California Institute of Technology, Pasadena, CA 91125, USA \\ ${ }^{4}$ School of Physics \& Astronomy, Cardiff University, Cardiff CF243AA, UK \\ 5 Jet Propulsion Laboratory, California Institute of Technology, 4800 Oak Grove Drive, Pasadena, CA 91109-8001, USA \\ ${ }^{6}$ National Radio Astronomy Observatory, Charlottesville, VA 22903, USA \\ ${ }^{7}$ Department of Physics and Astronomy, MS 111, University of Toledo, 2801 W. Bancroft St., Toledo, OH 43606-3328, USA \\ Received 2012 March 26; accepted 2013 January 27; published 2013 February 14
}

\begin{abstract}
The Wide-field Infrared Survey Explorer has revealed a T8.5 brown dwarf (WISE J111838.70+312537.9) that exhibits common proper motion with a solar-neighborhood (8 pc) quadruple star system- $\xi$ Ursae Majoris. The angular separation is 8.5 , and the projected physical separation is $\approx 4000 \mathrm{AU}$. The sub-solar metallicity and low chromospheric activity of $\xi$ UMa A argue that the system has an age of at least 2 Gyr. The infrared luminosity and color of the brown dwarf suggests the mass of this companion ranges between 14 and $38 M_{\mathrm{J}}$ for system ages of 2 and 8 Gyr, respectively.
\end{abstract}

Key words: brown dwarfs - infrared: stars - solar neighborhood - stars: late-type - stars: low-mass

\section{INTRODUCTION}

The effective temperature and thus spectrum of a brown dwarf evolves with time as it cools as a degenerate object (Kumar 1962). For an isolated brown dwarf, determination of mass and age are intertwined, such that a broad locus of mass and age will be consistent with a single measured effective temperature (Burrows et al. 2003). In rare instances brown dwarfs may reside in close binary systems, resolving this ambiguity with a direct dynamical mass estimation (Konopacky et al. 2010; Cardoso et al. 2009). In the absence of a dynamically measured mass, spectral modeling can significantly constrain a brown dwarf's mass if there exists sufficient restriction on the object's age. Constraints on age and metallicity are available if the brown dwarf is a member of a multiple star system. In this case, the properties of the primary, particularly chromospheric activity and kinematics, provide an indication of age.

The Wide-field Infrared Survey Explorer mission (WISE; Wright et al. 2010) has been a productive engine for the discovery of the coolest brown dwarfs. The WISE $W 1(3.4 \mu \mathrm{m})$ and $W 2(4.6 \mu \mathrm{m})$ filters are optimally tuned to select the coolest candidates, specifically those with spectra significantly shaped by methane absorption at low effective temperature (Mainzer et al. 2005). To date spectroscopic follow-up of WISE-selected sources has revealed more than 100 ultra-cool brown dwarfs (Kirkpatrick et al. 2011) including several exceptionally cool Y-dwarfs (Cushing et al. 2011; Kirkpatrick et al. 2012; Tinney et al. 2012), demonstrating that WISE colors provide for reliable photometric selection of ultra-cool brown dwarf candidates.

WISE J111838.70+312537.9, hereafter WISE 1118+31, easily meets the WISE brown dwarf color selection criterion (Kirkpatrick et al. 2011) with a $W 1-W 2$ color of 2.85 compared to a selection threshold of 2.0. Few confusing objects meet these selection restrictions. In addition this source lies 8'.5 from one of the nearer stars to the Sun, $\xi$ UMa, prompting an investigation into a possible system membership. This paper reports the spectral characterization of WISE $1118+31$ and the analysis of a series of astrometric observations spanning 26 months aimed at determining whether this source exhibits common proper motion with $\xi$ UMa. These observations demonstrate that WISE $1118+31$ is a newly found member of this already remarkable multiple star system. The characteristics of the primary system provide an indication of the metallicity and age of the newly discovered ultra-cool brown dwarf constraining the mass of this object.

\section{OBSERVATIONS}

\subsection{WISE}

WISE imaged the region of the sky containing WISE $1118+31$ on 20 occasions between 2010 May 21 04:20 UT and 2010 May 25 09:39 UT. The WISE All-sky Catalog reports this source as well detected in $W 1$ and $W 2$, with marginal signal at the position of the $W 1 / 2$ source in $W 3(12 \mu \mathrm{m})$ and only an upper limit in $W 4(22 \mu \mathrm{m})$. Of the 20 apparitions, 18 were sufficiently separated from a detector edge to permit source extraction. In all 18 cases the source was detected in $W 2$, producing a combined $\mathrm{S} / \mathrm{N}=34$ detection with $W 2=13.31$. Because exceptionally cool brown dwarfs are considerably fainter in $W 1$, which was optimized to produce a substantial flux difference between $W 1$ and $W 2$, WISE $1118+31$ is detected in $W 1$ in only 12 of the 18 opportunities with a combined $\mathrm{S} / \mathrm{N}=15$ and $W 1=16.16$, yielding a color of $W 1-W 2=2.85$. Figure 1 shows portions of the WISE image atlas covering both WISE $1118+31$ and $\xi \mathrm{UMa}$.

\subsection{Follow-up Imaging}

Multiple epochs of near-infrared imaging provide the astrometric data for WISE $1118+31$ needed to confirm common proper motion with $\xi$ UMa. Photometric information from these images is summarized in Table 1 while the astrometric data are listed in Table 2. No corrections for nonlinearity were applied to the ground-based photometry because the sky is brighter (Sánchez et al. 2008) than both WISE 1118+31 and the faint 

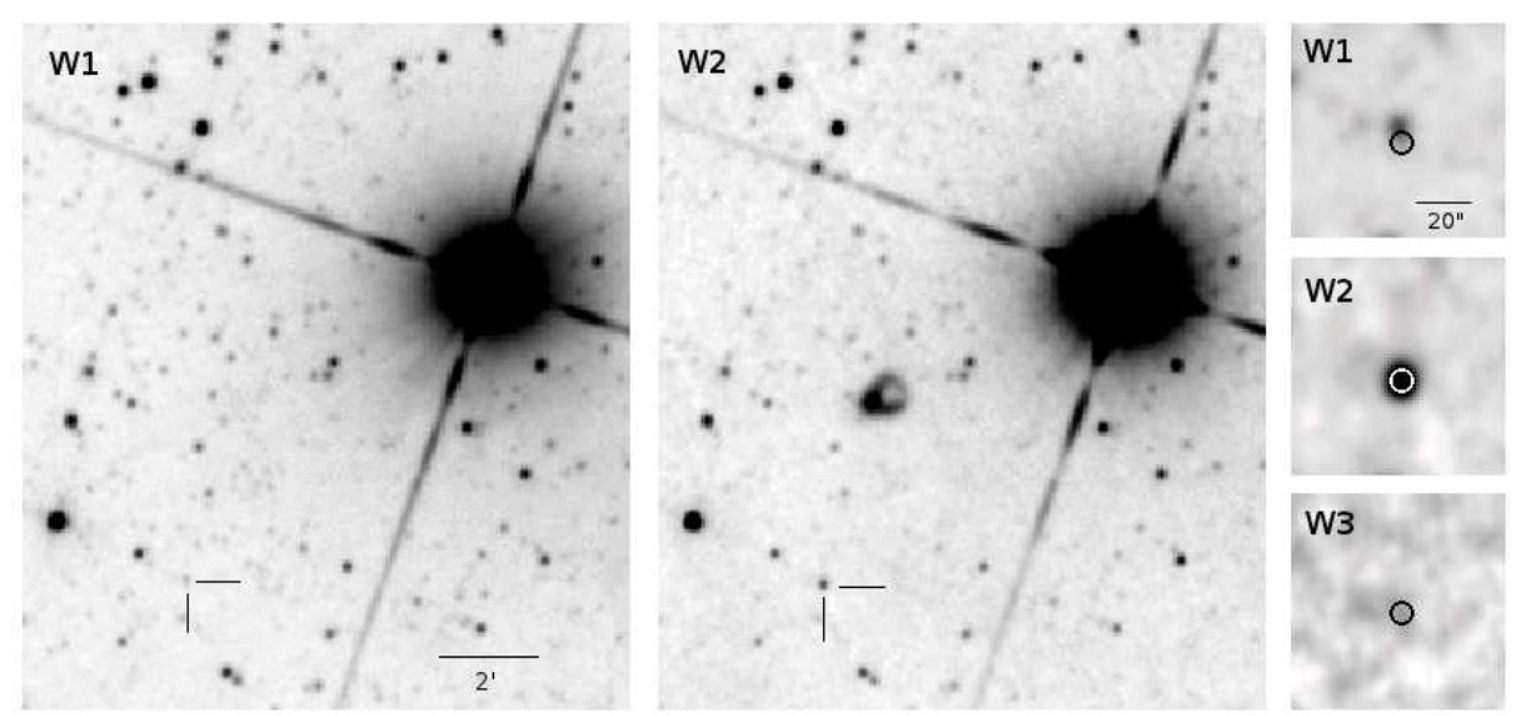

Figure 1. WISE All-sky Image Atlas cutouts showing a 10' FOV centered halfway between WISE 1118+31 (lower left) and $\xi$ UMa (upper right). The left panel shows $W 1(3.4 \mu \mathrm{m})$, while the right panel shows $W 2(4.6 \mu \mathrm{m})$. The lines point to WISE $1118+31$. The faint source near the lines in the left panel is the nearby $2 \mathrm{MASS}$ star, $\# 1$ on Figure 2. The odd shape above WISE $1118+31$ in the W2 image is a ghost image of $\xi$ UMa. The rightmost column shows $1^{\prime}$ postage stamps of WISE images at $3.4,4.6$, and $12 \mu \mathrm{m}$.

Table 1

Photometric Observations of WISE $1118+31$

\begin{tabular}{lcl}
\hline \hline Filter & Vega Magnitude & Instrument \\
\hline$Y(\mathrm{MKO})$ & $19.18 \pm 0.12$ & FanCam \\
$J(\mathrm{MKO})$ & $17.792 \pm 0.053$ & WHIRC \\
$J$ & $18.22 \pm 0.16$ & Bigelow \\
$J$ & $18.37 \pm 0.08$ & FanCam \\
$H(\mathrm{MKO})$ & $18.146 \pm 0.060$ & WHIRC \\
$H$ & $18.13 \pm 0.23$ & Bigelow \\
$K_{S}(\mathrm{MKO})$ & $18.746 \pm 0.150$ & WHIRC \\
$W 1$ & $16.160 \pm 0.071$ & WISE \\
ch1 & $15.603 \pm 0.026$ & IRAC \\
ch2 & $13.368 \pm 0.018$ & IRAC \\
$W 2$ & $13.308 \pm 0.032$ & WISE \\
$W 3$ & $12.359 \pm 0.314$ & WISE \\
$W 4$ & $>8.821$ & WISE \\
\hline
\end{tabular}

Notes. All magnitudes are Vega magnitudes and use the 2MASS $J H K_{s}$ filters except as noted. The $Y$-band calibration uses the Hamuy et al. (2006) transformation of $Y-K_{s}$ versus $J-K_{s}$.

Table 2

Astrometric Observations of WISE 1118+31

\begin{tabular}{lcccc}
\hline \hline Date & $\Delta \alpha \cos \delta$ & $\begin{array}{c}\Delta \delta \\
\left(^{\prime \prime}\right)\end{array}$ & Observatory & Band \\
& $-0.306 \pm 0.162$ & $-0.444 \pm 0.182$ & WISE & $W$ \\
2010.39 & $-0.165 \pm 0.211$ & $-0.603 \pm 0.191$ & FanMt & $Y$ \\
2010.91 & $-0.208 \pm 0.162$ & $-0.477 \pm 0.184$ & WISE & $W$ \\
2010.91 & $-0.232 \pm 0.067$ & $-0.794 \pm 0.067$ & Spitzer & ch2 \\
2011.05 & $-0.414 \pm 0.234$ & $-0.600 \pm 0.194$ & FanMt & $J$ \\
2011.12 & $-0.393 \pm 0.113$ & $-0.765 \pm 0.100$ & FanMt & $J$ \\
2011.24 & $-0.651 \pm 0.086$ & $-0.829 \pm 0.084$ & MtBglw & $J$ \\
2011.38 & $-0.554 \pm 0.194$ & $-0.723 \pm 0.190$ & MtBglw & $H$ \\
2011.38 & $-0.639 \pm 0.190$ & $-1.280 \pm 0.170$ & FanMt & $J$ \\
2011.85 & $-0.650 \pm 0.005$ & $-1.253 \pm 0.004$ & WIYN & $J$ \\
2012.00 & $-0.676 \pm 0.010$ & $-1.235 \pm 0.012$ & WIYN & $H$ \\
2012.00 & $-0.766 \pm 0.037$ & $-1.239 \pm 0.038$ & WIYN & $K$ \\
2012.00 & $-0.850 \pm 0.165$ & $-1.339 \pm 0.153$ & FanMt & $Y$ \\
2012.20 & $-0.695 \pm 0.117$ & $-1.631 \pm 0.126$ & Spitzer & ch2 \\
2012.15 & $-1.081 \pm 0.136$ & $-1.611 \pm 0.139$ & Spitzer & ch2 \\
2012.52 & & & & \\
\hline
\end{tabular}

Notes. Offsets relative to $11^{\mathrm{h}} 18^{\mathrm{m}} 38^{\mathrm{s}} .69+31^{\circ} 25^{\prime} 37^{\prime \prime} .7$ (J2000). Reference star: $11^{\mathrm{h}} 18^{\mathrm{m}} 38^{\mathrm{s}} .77+31^{\circ} 25^{\prime} 44^{\prime \prime} .2$ (2MASS).
Two Micron All Sky Survey (2MASS) objects used as calibrators. The absolute calibration uncertainties of $3 \%$ for Spitzer (Reach et al. 2005) and 2.4\%, 2.8\%, 4.5\%, and 5.7\% for W1..4 (WISE Explanatory Supplement Section IV.4.h.v) relative to Spitzer have not been included in the errors quoted in Table 1.

\subsubsection{Fan Mountain Observatory/FanCam}

$Y$ and $J$ photometry and astrometry of WISE $1118+31$ were obtained at various epochs between 2010 November 28 and 2012 March 15 with FanCam, a HAWAII-1 based near-infrared imager operating at the University of Virginia's Fan Mountain 31 inch telescope (Kanneganti et al. 2009). The source position was dithered by approximately $10^{\prime \prime}$ between $30 \mathrm{~s}$ exposures comprising total exposure times ranging from 60 to 80 minutes. The FanCam field of view (FOV) is 8.7 $\left(0^{\prime} .51 \mathrm{pixel}^{-1}\right)$. The central $7^{\prime} \times 7^{\prime}$ (Figure 2 ) of the combined, dithered exposures was fully covered, providing several 2MASS stars for photometric and astrometric reference. A median sky frame was subtracted from each individual exposure prior to flat fielding with the median background level subsequently restored to the image. $Y$ and $J$ aperture photometry was computed using an aperture with a radius of 3 pixels. The zero points for the $J$-band were computed using stars in the FOV with measured 2MASS magnitudes since the $J$-band filter in FanCam is based on the 2MASS system. In order to derive the $Y$-band zero point, we first computed the $Y$-band magnitudes of stars in the FOV using their 2MASS $J$ and $K_{s}$ magnitudes and the transformation given by Hamuy et al. (2006). The final uncertainties in the magnitudes include the photon noise from the sky and source, the read noise, and the uncertainty in the zero point due to the computed $Y$-band magnitudes of the calibrators. The resulting magnitudes and uncertainties are given in Table 1.

\subsubsection{Mount Bigelow/2MASS}

The former 2MASS camera on the $1.54 \mathrm{~m}$ Kuiper Telescope on Mt. Bigelow, AZ, has three $256 \times 256$ pixel NICMOS3 arrays simultaneously observing in 2MASS $J, H$, and $K_{s}$ filters (Milligan et al. 1996). The plate scale for all three arrays is $1^{\prime \prime} .65$ pixel $^{-1}$, resulting in a $7^{\prime}$ FOV. Exposures of $10 \mathrm{~s}$ duration, 


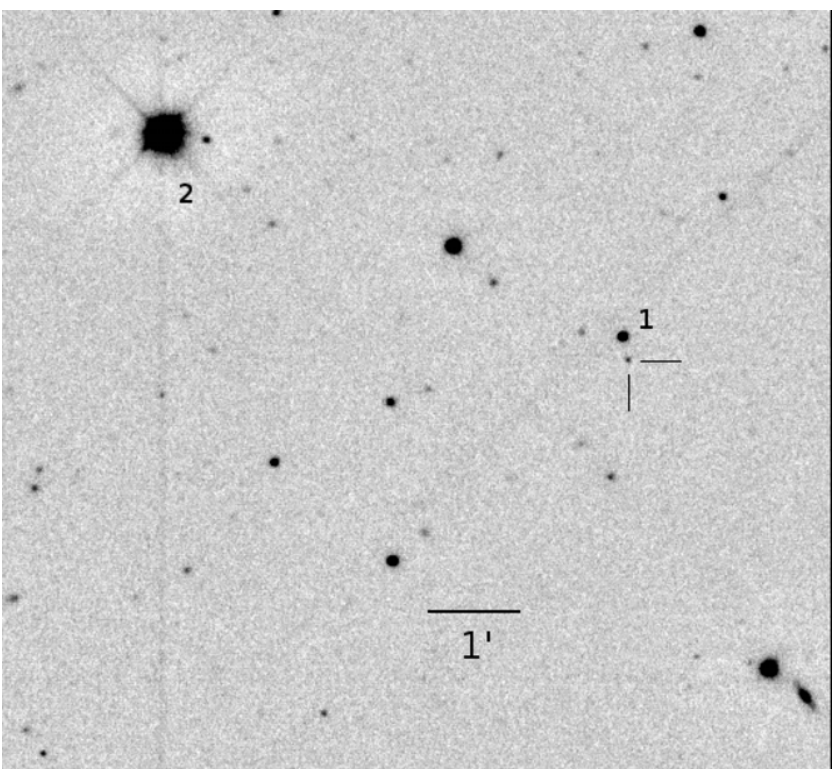

Figure 2. $Y$-band image of the WISE 1118+31 field obtained at Fan Mountain Observatory 2012 March 15 UT. North is up and east is to the left. Perpendicular lines mark the position of WISE $1118+31$. Star 1 nearby is the $J=15.94$ star 2MASS J11183876+3125441, while star 2 is 2MASS J11185086+3126520 with $J=10.32$. The faintest detected objects have $Y \approx 20$. The faint diffraction spike entering from the upper right (northwest) is from $\xi$ UMa 8.5 away.

216 in all, of WISE $1118+31$ were obtained on 2011 May 20 using six repeats of a $3 \times 3$ box dither pattern, with four consecutive images taken at each of the nine dither positions. The data were reduced using custom IDL routines implementing standard near-infrared flat fielding, background removal, and coaddition techniques. Flat fields in each band were constructed using on-source frames. 2MASS stars provided photometric reference in all three bands, leading to the magnitudes reported in Table 1.

\subsubsection{WIYN/WHIRC}

$J H K_{s}$ broadband imaging of WISE $1118+31$ was obtained on UT 2011 December 31 with the WIYN High-Resolution Infrared Camera (WHIRC; Meixner et al. 2010) and the WIYN $3.5 \mathrm{~m}$ Observatory. The data quality is excellent: both seeing ( $\sim 0^{\prime} .5$ with 0 '. 1 pixel scale) and photometric stability conditions were optimal. For each band, individual frames had exposure times of 120,120 , and $40 \mathrm{~s}$, for $J, H$, and $K_{s}$, respectively. WHIRC uses MKO filters for $J H K_{s}$. Using an efficient on-array dither pattern, a total of 7, 9, and 43 frames for $J, H$, and $K_{s}$, respectively, were obtained, thus providing a total exposure time of 840,1080 , and $1720 \mathrm{~s}$ for the $J, H$, and $K_{s}$ mosaics, respectively.

Individual frames were corrected for pupil-ghosts, dark and median sky flat subtracted, normalized by dome flat, and distortion corrected using information from the WHIRC user information guide. Astrometric and photometric solutions using 2MASS standards were then found. The fully reduced frames were combined into a deep mosaic, with outlier (bad pixel) rejection applied using temporal statistics. A final astrometric and flux calibration was then applied to the deep mosaic. The photometric uncertainty (comparing with the 2MASS PSC) was typically better than $5 \%$ for each mosaic produced. The achieved spatial resolution was $\sim 0$ '. $^{\prime}-0$ '.7 for the final mosaics, and the astrometric uncertainty was $\sim 0$ ! 05 . The target source, WISE $1118+31$, was detected in all three bands. Using a 1 .' 1 radius circular aperture, the background-subtracted integrated (Vega) magnitudes found are reported in Table 1. Note that WHIRC uses MKO filters, and the expected difference $J_{2 \mathrm{MASS}}-J_{\mathrm{MKO}}$ for a T8.5 brown dwarf is $\approx 0.36$ mag (Stephens \& Leggett 2004). If we correct the $J_{2 \text { MASS }}$ values by this amount we get three values for $J_{\mathrm{MKO}}: 17.79,17.86$, and 18.01 . The mid-range of these values is $J_{\mathrm{MKO}}=17.9$, which we adopt, giving $J_{\mathrm{MKO}}-W 2=4.6$.

\subsubsection{Spitzer}

The Infrared Array Camera (IRAC; Fazio et al. 2004) on board the Spitzer Space Telescope employs $256 \times 256$ pixel detector arrays to image a FOV of $5.2 \times 5 ! 2\left(1^{\prime \prime} .2 \mathrm{pixel}^{-1}\right)$. IRAC was used during the warm Spitzer mission to obtain deeper photometry in its 3.6 and $4.5 \mu \mathrm{m}$ channels (hereafter ch1 and ch2, respectively) than WISE was able to take in its $W 1$ and $W 2$ bands. These observations were made as part of Cycle 7 and Cycle 8 programs 70062 and 80109 (PI: Kirkpatrick). Our standard data acquisition and reduction methodology for IRAC observations is outlined in Kirkpatrick et al. (2011).

\subsubsection{Keck Adaptive Optics Imaging}

High resolution imaging observations of WISE 1118+31 were obtained using the Keck II LGS-AO system (Wizinowich et al. 2006; van Dam et al. 2006) with NIRC2 on 2012 April 14 (UT). WISE $1118+3125$ is not bright enough to serve as the tip-tilt reference star for the LGS-AO system, so we used the nearby 2MASS star (\#1 on Figure 2), which has $R=16.7$ and is located about $7^{\prime \prime}$ from the target. The seeing was generally very good throughout the night $\left(0{ }^{\prime \prime} 3-0,5\right)$; however, high clouds were present during the observations of WISE $1118+31$. We used the MKO $H$ filter and narrow plate scale $\left(00^{\prime} .009942\right.$ pixel $^{-1}$ for a single-frame FOV of $\left.10^{\prime \prime} \times 10^{\prime \prime}\right)$ for the observations. The data were obtained by using a three-point dither pattern that avoided the noisy, lower left quadrant of the array. Each image had an integration time of $120 \mathrm{~s}$ and the dither pattern was repeated five times to give a total exposure time of $1800 \mathrm{~s}$.

The images were reduced in a standard fashion using custom IDL scripts. These steps included dark frame subtraction, flat fielding (using a dome flat), and sky subtraction from a sky frame created from the dithered science frames. The individual frames were then shifted to move WISE $1118+31$ to the center of the array and the stack was median averaged to create the final mosaic seen in Figure 3. The FWHM in the final mosaic is 54 mas and shows no irregularities or evidence for a close companion.

\subsection{Spectroscopy \\ 2.3.1. LBT-LUCIFER}

We obtained an $H$ - and $K$-band spectrum of WISE $1118+31$ on 2012 December 12 (UT) using the Large Binocular Telescope (LBT) Near-Infrared Spectroscopic Utility with Camera and Integral Field Unit for Extragalactic Research (LUCIFER; Mandel et al. 2008). A series of twelve 300 s exposures was obtained at different positions along the $4^{\prime}$ slit to facilitate sky subtraction. The A0 V star HD 97034 was also observed for telluric correction and flux calibration purposes. A series of halogen lamp exposures were also obtained for flat fielding purposes.

The data were reduced using custom Interactive Data Language (IDL) software based on the Spextool data reduction package (Cushing et al. 2004). Pairs of images taken at two different positions along the slit were first subtracted and flat-fielded. The spectra were then extracted and wavelength 


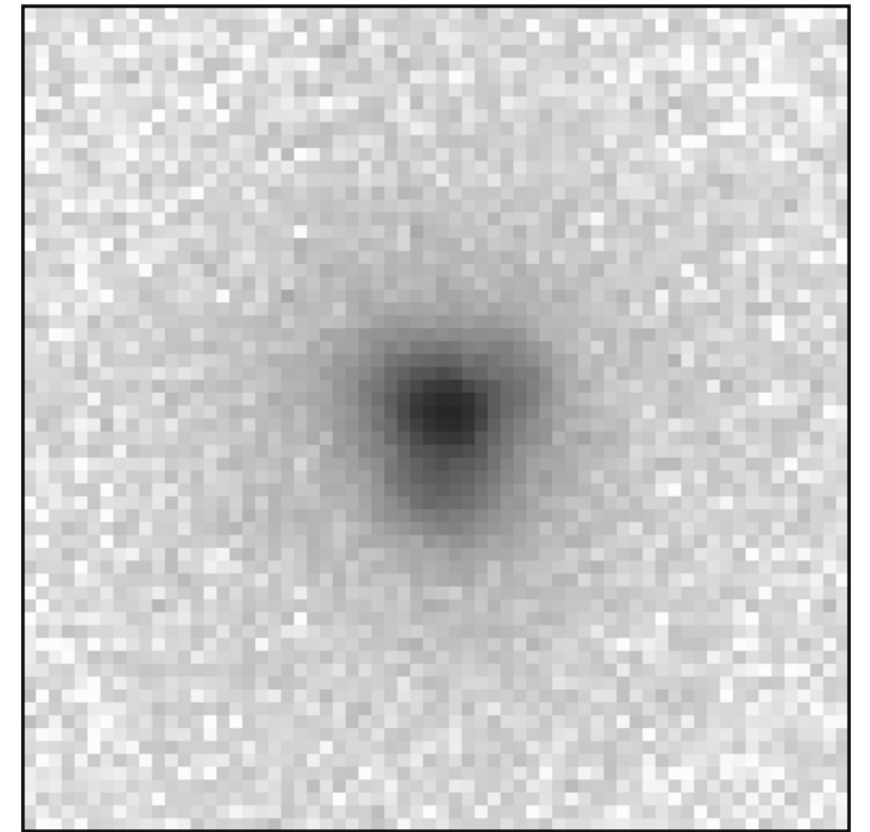

Figure 3. $H$-band LGS-AO image of WISE $1118+3125$ taken with the NIRC2 camera on Keck II. The image is $\approx 0^{\prime} .6$ on a side with north up and east to the left. There is no evidence for an equal brightness companion beyond a separation of 50 mas.

calibrated using sky emission lines of $\mathrm{OH}$ and $\mathrm{CH}_{4}$. The twelve spectra are combined and then corrected for telluric absorption and flux calibrated using the technique described by Vacca et al. (2003). The final spectrum is shown in Figure 4.

\subsubsection{Hale-TripleSpec}

A 1-2.5 $\mu \mathrm{m}$ spectrum of WISE $1118+31$ was obtained with the Triple Spectrograph (TripleSpec; Herter et al. 2008) at the $5.08 \mathrm{~m}$ Hale Telescope at Palomar Observatory. The 1-2.5 $\mu \mathrm{m}$ range is covered over four cross-dispersed orders which are imaged simultaneously on the $1024 \times 2048$ HAWAII-2 array. The $1^{\prime \prime}$ wide slit provides a resolving power of $R \approx 2700$. A series of eight, $300 \mathrm{~s}$ exposures were obtained at two different positions along the $30^{\prime \prime}$ long slit to facilitate sky subtraction. The A0 V star HD 99966 was observed for telluric correction and flux calibration purposes and dome flats were obtained at the start of the night.

The data were reduced using a modified version of the Spextool (Cushing et al. 2004) package; a detailed description of the reduction steps can be found in Kirkpatrick et al. (2011). Briefly, a two-dimensional wavelength solution is derived using sky emission features of $\mathrm{OH}$ and $\mathrm{CH}_{4}$. Spectra are then extracted from pair-subtracted, flat-fielded images. The resulting spectra are combined and corrected for telluric absorption and flux calibrated on an order-by-order basis. Finally, the spectra from each order are stitched together to form a complete $1-2.5 \mu \mathrm{m}$ spectrum. The spectrum was then flux calibrated as described in Rayner et al. (2009) using the photometry in Table 1. The final spectrum is shown in Figure 4.

\section{DISCUSSION}

\subsection{Properties of the Central Star System}

$\xi$ Ursae Majoris $^{8}$ is a complex stellar system with at least four, and possibly five, components (Mason et al. 1995) known

\footnotetext{
Also Alula Australis, HR 4374/5, Gl 423, HD 98230/1.
}

prior to the discovery of WISE $1118+31$. Visible to the unaided eye a short distance from the Big Dipper, this telescopic double star was among the first to be recognized as a gravitationally bound binary system (Herschel 1804). Given the $60 \mathrm{yr}$ period of the visual pair it was not until 23 yr later that Struve (1827) calculated a formal orbit. Subsequently both components of the visual pair with $a=2$ ".536 (Mason et al. 1995) were found to be spectroscopic binaries. Heintz (1967) finds periods of 669.1 days for the Aa system and 3.9805 days for the Bb system. Griffin (1998) gives velocity amplitudes, $K_{\mathrm{Aa}}=4.85 \pm 0.14 \mathrm{~km} \mathrm{~s}^{-1}$ and $K_{\mathrm{Bb}}=4.33 \pm 0.09 \mathrm{~km} \mathrm{~s}^{-1}$ in the $60 \mathrm{yr}$ orbit which lead to a dynamical parallax estimate of $0.126 \pm 0.0023$.

However Söderhjelm (1999) re-analyzed the Hipparcos data combined with other data for visual binaries and gives 0'.1197士 0 '.0008 for the parallax of $\xi \mathrm{UMa}$, and total mass for the $\mathrm{AaBb}$ system of $2.62 M_{\odot}$.

The weighted mean of the dynamical parallax and the Hipparcos parallax is $0^{\prime \prime} .1206 \pm 0^{\prime \prime} 00074$ but with $\chi^{2}=8.6$ for 1 degree of freedom in the fit for the mean, so we inflate the errors by a factor of $\sqrt{8.6}$ and adopt $0.1206 \pm 0.0022$ for the parallax.

Since the possible fifth component, seen by Mason et al. (1995) separated by 56 milliarcseconds from the B component of the visual binary, was only detected at one epoch we will not consider it to be a member of the system.

Bakos et al. (2002) give a proper motion of $0.75 \mathrm{yr}^{-1}$ in position angle 217.49 , based on a $29 \mathrm{yr}$ interval. These values resolve into $-0.456 \mathrm{yr}^{-1}$ in R.A. and $-0.595 \mathrm{yr}^{-1}$ in decl., which we adopt.

\subsubsection{Spectral Types and Metallicities of the Central Stars}

Cayrel de Strobel et al. (1994) used high-resolution spectroscopy to find effective temperatures and gravities of $5950 \pm 30 \mathrm{~K}, \log g=4.3 \pm 0.2$ for $\xi \mathrm{UMa} \mathrm{A}$; and $5650 \pm 50$ $\mathrm{K}, \log g=4.5 \pm 0.2$ for $\mathrm{B}$. These temperatures and gravities are consistent with spectral types of F8.5V and G2V assigned to $\xi$ UMa A and B by Keenan \& McNeil (1989). Cayrel de Strobel et al. (1994) found that both stars had slightly sub-solar iron abundances, $[\mathrm{Fe} / \mathrm{H}]=-0.32 \pm 0.05 \mathrm{dex}$.

\subsubsection{Chromospheric Activity and Age of the Central Stars}

Cayrel de Strobel et al. (1994) see chromospheric calcium emission lines for $\xi$ UMa B but not for $\xi$ UMa A. They propose that the short four-day period orbit of the $\mathrm{Bb}$ system is driving the chromospheric activity in B. From the lack of emission in A, Cayrel de Strobel et al. (1994) conclude that $\xi$ UMa is older than stars in the cluster NGC 752, which has an age of 2 Gyr. Ball et al. (2005) report X-ray observations of $\xi$ UMa which show that all of the observed X-ray emission is coming from the fourday period binary $\mathrm{Bb}$. X-ray flux from component $\mathrm{A}$ is more than two orders of magnitude fainter, implying $\log L_{X}<27.5$. Since component $\mathrm{A}$ is close to solar luminosity, the ratio of X-ray to bolometric flux is $R_{X}=\log \left(L_{X} / L_{\text {bol }}\right)<-6$ which gives an age greater than 4 Gyr using Equation (A3) in Mamajek \& Hillenbrand (2008). The low space velocity $(U, V, W)=(-2.36 \pm 0.33,-28.45 \pm 1.22,-20.26 \pm$ $0.25) \mathrm{km} \mathrm{s}^{-1}$ calculated by Karataş et al. (2004) implies that $\xi$ UMa is a member of the thin disk, so ages much greater than 8 Gyr are unlikely. Finally Cayrel de Strobel et al. (1994) found that the luminosities and effective temperatures were consistent within the errors with the masses derived by Heintz (1967) for a 5 Gyr isochrone calculated with sub-solar abundance. 


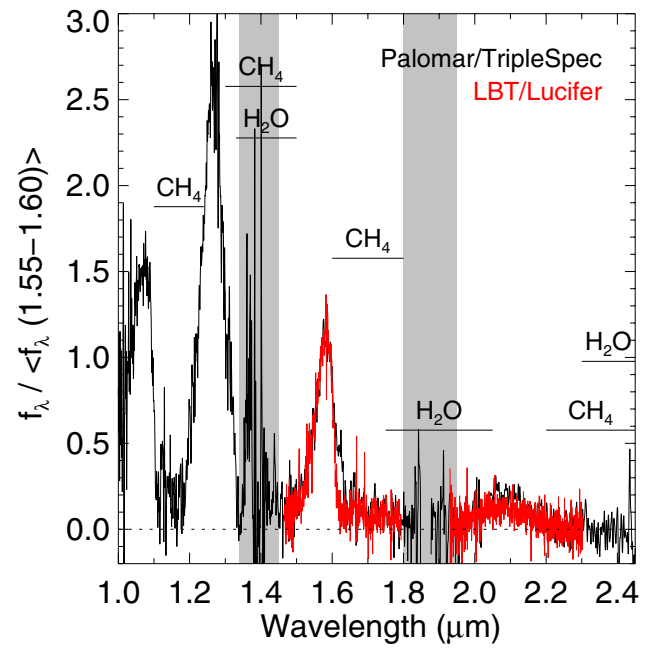

Figure 4. Spectrum of WISE 1118+31 obtained with TripleSpec (black) and LUCIFER (red). Regions of strong telluric absorption are shown in gray. Prominent absorption bands of $\mathrm{CH}_{4}$ and $\mathrm{H}_{2} \mathrm{O}$ are indicated. The agreement between the two spectra is excellent.

\subsection{Astrometry of the Companion}

Astrometric information was extracted from the observed images at the various epochs using the standard maximum likelihood technique in which a point-spread function (PSF) was fit to each source profile. The positional uncertainties were estimated using an error model which includes the effects of instrumental and sky background noise and PSF uncertainty. The PSF and its associated uncertainty map were estimated for each image individually using a set of bright stars in the field. In order to minimize systematic effects, our astrometry was based on relative positions, using as a reference the nearby star 2MASS $\mathrm{J} 11183876+3125441$ at a separation of approximately 6.'5 (star 1 in Figure 2). This is sufficiently close that systematic errors due to such effects as focal-plane distortion, plate scale, and rotation errors cancel out in the relative position to an accuracy much greater than the random estimation errors. We do, however, assume that the proper motion and parallax of the reference star are negligible compared to those of WISE $1118+31$. In support of this assumption, we find no significant difference in relative motion of WISE $1118+31$ when the differential astrometry is repeated using more distant 2MASS reference stars, at separations of $69^{\prime \prime}$ and $105^{\prime \prime}$. The astrometric estimation procedure is discussed in more detail by Marsh et al. (2013).

Table 2 gives the measured position offsets which were input to a parallax and proper motion code that handles both Earthbased and Spitzer observations. Table 3 gives the output from the code for three different sets of free parameters. In one case the proper motion and parallax were forced to zero. In the second case they were forced to match $\xi$ UMa. In the final case the proper motion and parallax were left as free parameters.

The astrometric fits for WISE $1118+31$ are a very good match to the motion of $\xi$ UMa. The $\Delta \chi^{2}=241.0$ between the best fit and a fit with a fixed position shows that the motion of WISE $1118+31$ has been detected with an $\mathrm{S} / \mathrm{N}$ of 15.5 , while the $\Delta \chi^{2}=1.28$ for 3 extra degrees of freedom between the best fit and a fit forced to match the proper motion and parallax of $\xi$ UMa is perfectly consistent with WISE $1118+31$ being a bound member of the $\xi$ UMa system. Figure 5 shows two fits: one forced to match $\xi$ UMa and one with the proper motion and parallax as free parameters.

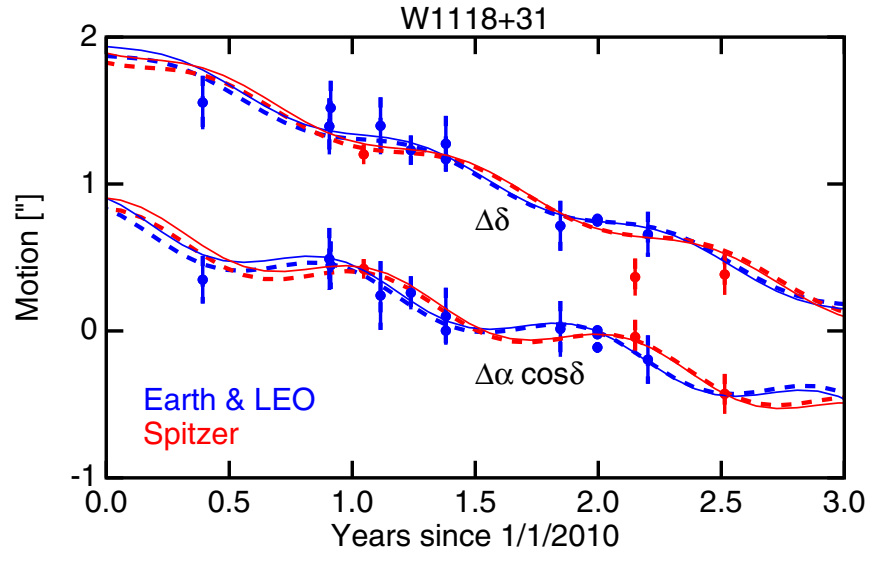

Figure 5. Astrometric data and fits for WISE 1118+31. The blue curves and points are for ground-based or Low Earth Orbit observatories, while the red curves and points are for Spitzer. The bold dashed lines show the fit with proper motion and parallax as free parameters, while the lighter solid curves show the fit forced to match $\xi$ UMa. These fits are very similar. Note that $\Delta \delta$ has been displaced by a constant for clarity.

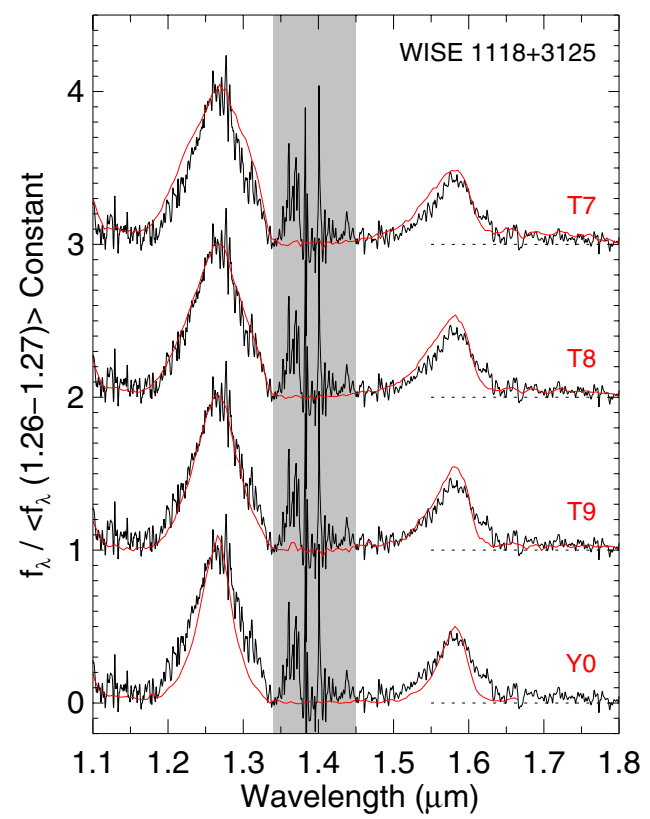

Figure 6. Sequence of spectral standards from T7 to Y0 (red) along with the spectrum WISE $1118+31$ (black). The spectral standards are 2MASS 0727+1710 (T7; Burgasser et al. 2006, 2002) and 2MASS 0415-0935 (T8; Burgasser et al. 2006, 2002), UGPS 0722-05 (T9; Cushing et al. 2011; Lucas et al. 2010), and WISE 1738+2732 (Y0; Cushing et al. 2011). The spectrum of WISE $1118+3125$ has been smoothed to a resolving power of $\lambda / d \lambda=1000$ for display purposes. Spectra are normalized to unity over the $1.26-1.27 \mu \mathrm{m}$ wavelength range and offset for clarity (dotted lines). Regions of high telluric absorption are shown in gray. WISE $1118+31$ is classified as T8.5 \pm 0.5 . The mismatch between the spectrum of WISE $1118+31$ and the templates at $\sim 1.63 \mu \mathrm{m}$ is due to poor subtraction of the $\mathrm{OH}$ sky lines.

\subsection{The Nature of the Companion \\ 3.3.1. Spectral Classification}

As shown in Figure 4, the spectrum of WISE 1118+31 exhibits deep absorption bands of $\mathrm{CH}_{4}$ and $\mathrm{H}_{2} \mathrm{O}$ indicative of $\mathrm{T}$ dwarfs. We derive a more precise spectral type using the spectral classification schemes of Burgasser et al. (2006) and the extension to this system by Cushing et al. (2011) whereby UGPS J072227.51-054031.2 (hereafter UGPS 0722-05; Lucas et al. 2010) is defined as the T9 spectral standard and WISE 
Table 3

Astrometric Fits for WISE 1118+31

\begin{tabular}{lrccc}
\hline \hline Type & $\chi^{2}$ & \#df & $\begin{array}{c}\cos \delta d \alpha / d t \\
\left({ }^{\prime \prime} \mathrm{yr}^{-1}\right)\end{array}$ & $\begin{array}{c}d \delta / d t \\
\left(" \mathrm{yr}^{-1}\right)\end{array}$ \\
\hline Fixed & 270.836 & 28 & 0 & 0 \\
Forced to $\xi$ UMa & 29.856 & 28 & -0.456 & 0.595 \\
Free & 28.579 & 25 & $-0.419 \pm 0.048$ & $-0.563 \pm 0.045$
\end{tabular}

$1738+2732$ is defined as the Y0 spectral standard. As shown in Figure 6, WISE $1118+31$ has a spectral type of T8.5 based on the width of the $J$-band peak at $1.27 \mu \mathrm{m}$.

\subsubsection{Absolute Magnitude, Luminosity, and Mass}

For our adopted parallax the absolute magnitude is $M_{W 2}=$ $W 2+5 \log (10 \varpi)=13.715 \pm 0.051$. This agrees very well with the $M_{W 2}$ versus type relation in Kirkpatrick et al. (2011) which gives $M_{W 2}=13.71 \pm 0.21$ for a spectral type of T8.5 \pm 0.5 . The $M_{W 2}$ versus type relation in Kirkpatrick et al. (2012) with W1828+2650 excluded gives $M_{W 2}=13.64 \pm 0.31$ which also agrees with the observed $W 2$ flux. This $M_{W 2}=13.715$ implies $v L_{v}=1.14 \times 10^{-6} L_{\odot}$ at $4.6 \mu \mathrm{m}$.

To convert $v L_{v}$ to $L$ we need the factor $v F_{v}(W 2) / F_{\text {bol }}$, which is equivalent to the bolometric correction, since $v F_{v}=$ $\left(\lambda_{\text {iso }} F_{\lambda}^{\circ} / f_{c}\right) 10^{-0.4 m}$ (Wright et al. 2010) which is $1.118 \times$ $10^{-7} \mathrm{erg} \mathrm{cm}^{-2} \mathrm{~s}^{-1}$ for $W 2=0$ with a $v^{-1}$ spectrum, and $F_{\text {bol }}=2.48 \times 10^{-5} 10^{-0.4 m_{\text {bol }}} \mathrm{erg} \mathrm{cm}^{-2} \mathrm{~s}^{-1}$, so

$$
2.5 \log \left(\frac{v F_{v}(W 2)}{F_{\text {bol }}}\right)=-5.87+m_{\text {bol }}-m(W 2) \text {. }
$$

Thus

$$
\begin{aligned}
\mathrm{BC}(W 2) & =m_{\mathrm{bol}}-m(W 2) \\
& =2.5 \log \left(\frac{v F_{v}(W 2)}{F_{\mathrm{bol}}}\right)+5.87 .
\end{aligned}
$$

Note that $v F_{v} / F_{\text {bol }}$ is non-negative and also normalized since $\int\left(v F_{v} / F_{\text {bol }}\right) d \ln v=1$ by definition. Since the normalization integral is dominated by the brightest peaks, errors in the faint parts of the spectral energy distribution (SED) have only small effects on the derived value of $v F_{v}(W 2) / F_{\text {bol }}$ or equivalently the bolometric correction. The brightest peaks in $v F_{v} / F_{\text {bol }}$ for late T dwarfs are in the $J$ band and the $W 2$ band, so if we get the $J-W 2$ color right then our value of $\nu F_{\nu}(W 2) / F_{\text {bol }}$ will be fairly accurate.

We will use a model spectrum to evaluate $v F_{v}(W 2) / F_{\text {bol }}$ in order to fill in the gaps between the photometric passbands, but we are only using the shape of the spectrum to calculate the bolometric correction. Thus, even if the effective temperature $T_{\text {eff }}$ is a parameter of the model, that does not imply that the model parameter is the actual $T_{\text {eff }}$ of the star. y

Given a model spectrum $F_{v}$, the ratio $v F_{v}(W 2) / F_{\text {bol }}$ is given

$$
\frac{v F_{v}}{F_{\mathrm{bol}}}=\frac{\int\left(v F_{v} / h v\right) R(v) d \ln v}{\int\left(\left[1 \mathrm{erg} \mathrm{cm} \mathrm{cm}^{-2} \mathrm{~s}^{-1}\right] / h v\right) R(v) d \ln v \int F_{v} d v}
$$

where $R(v)$ is the response per photon (Wright et al. 2010). The model we use is a linear combination of sulfide cloud models from Morley et al. (2012) with $T_{\text {eff }}=600 \mathrm{~K}, \log (g)=5.0$. We form the sum of 0.2 times the flux from the cloudless model and 0.8 times the flux from the $f_{\text {sed }}=4$ model. Physically this is a brown dwarf that is $20 \%$ covered by clear zones and $80 \%$ covered by cloudy bands. This linear combination gives $v F_{v} / F_{\text {bol }}=1.46, J_{\mathrm{MKO}}-W 2=4.60,(Y-J)_{\mathrm{MKO}}=1.23$, $(J-H)_{\mathrm{MKO}}=-0.18,\left(J-K_{s}\right)_{\mathrm{MKO}}=-0.06$ and $W 1-W 2=$ 3.17 . The model matches the observed $J-W 2$ color by design but is too bright in $K_{s}$ and too faint in $W 1$. If we make an ad hoc correction to the SED by making the wavelength range from 3.0 to $3.8 \mu \mathrm{m}$ covering the $W 1$ band 0.4 mag brighter that would match the $W 1-W 2$ color and change the normalization integral by $2 \%$. If we make the wavelength range from 2.0 to $2.3 \mu \mathrm{m}$ covering the $K_{s}$ band $0.8 \mathrm{mag}$ fainter, then the $J-K_{s}$ color matches the data and the normalization integral changes by $-1.3 \%$. Thus we adopt $v F_{v}(W 2) / F_{\text {bol }}=1.46$ with a $10 \%$ uncertainty to allow for such errors in the model, giving $L=(1.14 / 1.46) \times 10^{-6}=10^{-6.107 \pm 0.043} L_{\odot}$ and $\mathrm{BC}(W 2)=6.28$ so $M_{\mathrm{bol}}=20.0 \pm 0.11$ for WISE $1118+31$. Interpolating the $L$ versus $M$ and $t$ figures in Saumon \& Marley (2008) gives this luminosity for masses of 14 to $31 M_{\text {J }}$ with ages of 2 and 8 Gyr using the cloudy models, and 17 to $38 M_{\mathrm{J}}$ for the cloudless models.

Even though the model parameters do not necessarily apply to the actual star, we find that they do give a mass and luminosity consistent with those derived above. The $T_{\text {eff }}=600 \mathrm{~K}, \log (g)=$ 5.0 models of Hubeny \& Burrows (2007) have radii of $0.91 R_{\mathrm{J}}$, and a mass $M=g R^{2} / G=32 M_{\mathrm{J}}$. The luminosity calculated directly from $T_{\text {eff }}$ and $R$ is $L=4 \pi R^{2} T_{\text {eff }}^{4}=10^{-6.01} L_{\odot}$ so this model is reasonably self-consistent. Calculating the temperature from $R$ and $L$ gives $T_{\text {eff }}=\left(0.91 R_{\mathrm{J}} / R\right)^{1 / 2}(567 \pm 14) \mathrm{K}$.

\subsection{Another Binary?}

If something like the Morley et al. (2012) sulfide clouds is not causing the red $J-W 2$ color of WISE $1118+31$, then there is a tension between the absolute magnitude $M_{W 2}$, which requires a fairly high $T_{\text {eff }}$, and the colors and spectral type which suggest a lower temperature. This could be ameliorated if WISE $1118+31$ were an equal mass binary which would increase the total radiating area. In this case the absolute magnitude of one component of the binary would be $M_{W 2}=14.47$ and the colors would remain the same. The best fitting Hubeny \& Burrows (2007) model is then a non-chemical equilibrium model with $T_{\text {eff }}=500 \mathrm{~K}$ and $\log (g)=5.0$. This model has $M_{W 2}=14.27, W 1-W 2=4.43, J(\mathrm{MKO})-W 2=$ 4.57 , and $R=0.888 R_{\mathrm{J}} \cdot v F_{v} / F_{\text {bol }}=1.546$ in the $W 2$ band for this model, so the luminosity of a single component is $L=0.5 \times 1.14 \times 10^{-6} / 1.546=3.7 \times 10^{-7} L_{\odot}$. The mass range for ages from 2 to 8 Gyr in the Saumon \& Marley (2008) cloudless models is 12 to $29 M_{\mathrm{J}}$, while the cloudy models give 10 to $25 M_{\mathrm{J}}$. Thus the range of total masses is 21 to $59 M_{\mathrm{J}}$ under the equal mass binary hypothesis, which is not much different than the range under the single object hypothesis. However, the absolute magnitude of the components would no longer agree with the $M_{W 2}$ versus type relation (Kirkpatrick et al. 2011, 2012). 
As stated in Section 2.2.5, we saw no evidence for binarity in the 2010 April 14 NIRC2 images of WISE 1118+31. To determine the upper limit on the separation of an equal brightness companion, we added a shifted copy of the mosaic to the original mosaic. At separations $\leqslant 4$ pixels, we are unable to convincingly establish the presence of the companion because of the difficulty in differentiating between an irregular PSF caused by an off-axis tip-tilt reference star and an elongated PSF caused by a companion when no other stars are present on the array. At separations of $\geqslant 5$ pixels $(\geqslant 50$ mas), the presence of a companion is obvious. Therefore, we set the upper limit on the separation to 50 mas, slightly less than the FWHM of the image. If this was not due to a temporary conjunction, so the actual separation were less than $0.4 \mathrm{AU}$, then the orbital period could be less than two years.

\subsection{The View from the Neighborhood of WISE 1118+31}

With a projected separation of $4100 \mathrm{AU}$ from the four main components of the $\xi$ UMa system, WISE $1118+31$ has a distant but interesting perspective. The two binaries that form the $2^{\prime \prime}$ visual pair as seen from the Earth are separated by $20 \mathrm{AU}$, or by an angular scale of $15^{\prime}$ from the distant perspective of WISE $1118+31$. At that distance the A and B components would each shine with an apparent visual magnitude of -9 , one hundred times brighter than Venus in the skies of Earth.

Given the complexity of the $\xi$ UMa system, it is reasonable to speculate that WISE $1118+31$ could be a component that was once more closely bound to the system, which was thrown into an orbit with a very large apocenter during a three-body interaction that tightened the $\xi$ UMa Bb binary. This would make WISE $1118+31$ similar in history to an Oort Cloud comet, formed at a radius of tens of AU from the central star system, lifted into an orbit with a high apocenter by multi-body perturbations, followed by galactic tides raising the pericenter (Duncan et al. 1987). Given an orbital period of the order of $10^{5} \mathrm{yr}$, the orbital eccentricity of WISE $1118+31$ may ultimately be detectable. With an apparent orbital radius of 8.5 the orbital motion will amount to tens of milliarcseconds per year relative to the primary system, and a radial velocity difference of about $1 \mathrm{~km} \mathrm{~s}^{-1}$. Characterizing this motion, specifically the differential proper motion between WISE $1118+31$ and its primary, is tractable given modern infrared and visual astrometric capability, and certainly will be accomplished with the passage of time.

\section{CONCLUSIONS}

Alula Australis ( $\xi$ UMa), a solar neighborhood visual binary where each component is itself a spectroscopic binary, possesses an ultra-cool brown dwarf (T8.5) companion at a projected separation of $4100 \mathrm{AU}$. This system is similar to, but somewhat more complex than, the binaries studied by Allen et al. (2012), who found that one in five spectroscopic binary systems had distant common proper motion companions. Faherty et al. (2010) found that wide companions were much more likely with binary or tertiary central objects, and $\xi$ UMa is a continuation of that trend. Thus the $\xi$ UMa system is uncommon primarily in the apparent magnitude of the central star system, due to its proximity to the Sun. The $\xi$ UMa system provides a very accurate absolute magnitude for a T8.5 brown dwarf, but both the age of the system and the mass of the brown dwarf are uncertain due to the difficulty of determining the age of a star in the middle of its main sequence lifetime. WISE $1118+31$ appears to be slightly less luminous and redder than the T8 dwarf WISEP J1423+01 which is a common proper motion companion to BD+01 2920 (Pinfield et al. 2012).

The $\xi$ UMa system is amenable to detailed study since it is quite close to the Sun. An accurate (sub $\mathrm{km} \mathrm{s}^{-1}$ ) radial velocity for WISE $1118+31$ would be valuable information for determining its orbit around $\xi$ UMa. Improved proper motions for both WISE $1118+31$ and the center of mass of the central quadruple star system are also needed for orbit characterization.

There is a tension between the red $J-W 2$ color of WISE $1118+31$ and its absolute magnitude $M_{W 2}$ when fitting to older models. This discrepancy could be relaxed if WISE $1118+31$ were a binary, but one AO observation showed no evidence for binarity. Thus the new types of clouds considered by Morley et al. (2012) may well be significant, and we conclude that model spectra need to be updated to fit the observations of WISE $1118+31$ and the many other late $\mathrm{T}$ and $\mathrm{Y}$ dwarfs found in the WISE data.

This publication makes use of data products from the Widefield Infrared Survey Explorer, which is a joint project of the University of California, Los Angeles, and the Jet Propulsion Laboratory/California Institute of Technology, funded by the National Aeronautics and Space Administration.

This work was partially supported by a NASA Keck PI Data Award, administered by the NASA Exoplanet Science Institute. Part of the data presented herein were obtained at the W. M. Keck Observatory from telescope time allocated to the National Aeronautics and Space Administration through the agency's scientific partnership with the California Institute of Technology and the University of California. The Observatory was made possible by the generous financial support of the W. M. Keck Foundation. The authors wish to recognize and acknowledge the very significant cultural role and reverence that the summit of Mauna Kea has always had within the indigenous Hawaiian community. We are most fortunate to have the opportunity to conduct observations from this mountain.

Facilities: WISE, FMO:31in (FanCam), LBT (LUCIFER), Hale (TripleSpec), SO:Kuiper (2MASS), WIYN (WIRC), Keck:II (NIRC2-LGSAO)

\section{REFERENCES}

Allen, P. R., Burgasser, A. J., Faherty, J. K., \& Kirkpatrick, J. D. 2012, AJ, 144,62

Bakos, G. Á., Sahu, K. C., \& Németh, P. 2002, ApJS, 141, 187

Ball, B., Drake, J. J., Lin, L., et al. 2005, ApJ, 634, 1336

Burgasser, A. J., Geballe, T. R., Leggett, S. K., Kirkpatrick, J. D., \& Golimowski, D. A. 2006, ApJ, 637, 1067

Burgasser, A. J., Kirkpatrick, J. D., Brown, M. E., et al. 2002, ApJ, 564, 421

Burrows, A., Sudarsky, D., \& Lunine, J. I. 2003, ApJ, 596, 587

Cardoso, C. V., McCaughrean, M. J., King, R. R., et al. 2009, in AIP Conf. Proc. 1094, Cool Stars, Stellar Systems and the Sun, ed. E. Stempels (Melville, NY: AIP), 509

Cayrel de Strobel, G., Cayrel, R., Friel, E., Zahn, J.-P., \& Bentolila, C. 1994, A\&A, 291, 505

Cushing, M. C., Kirkpatrick, J. D., Gelino, C. R., et al. 2011, ApJ, 743, 50

Cushing, M. C., Vacca, W. D., \& Rayner, J. T. 2004, PASP, 116, 362

Duncan, M., Quinn, T., \& Tremaine, S. 1987, AJ, 94, 1330

Faherty, J. K., Burgasser, A. J., West, A. A., et al. 2010, AJ, 139, 176

Fazio, G. G., Hora, J. L., Allen, L. E., et al. 2004, ApJS, 154, 10

Griffin, R. F. 1998, Obs, 118, 273

Hamuy, M., Folatelli, G., Morrell, N. I., et al. 2006, PASP, 118, 2

Heintz, W. D. 1967, AN, 289, 269

Herschel, W. 1804, RSPT, 94, 353

Herter, T. L., Henderson, C. P., Wilson, J. C., et al. 2008, Proc. SPIE, 7014, $70140 \mathrm{X}$

Hubeny, I., \& Burrows, A. 2007, ApJ, 669, 1248 
Kanneganti, S., Park, C., Skrutskie, M. F., et al. 2009, PASP, 121, 885 Karataş, Y., Bilir, S., Eker, Z., \& Demircan, O. 2004, MNRAS, 349, 1069 Keenan, P. C., \& McNeil, R. C. 1989, ApJS, 71, 245

Kirkpatrick, J. D., Cushing, M. C., Gelino, C. R., et al. 2011, ApJS, 197, 19

Kirkpatrick, J. D., Gelino, C. R., Cushing, M. C., et al. 2012, ApJ, 753, 156

Konopacky, Q. M., Ghez, A. M., Barman, T. S., et al. 2010, ApJ, 711, 1087

Kumar, S. S. 1962, AJ, 67, 579

Lucas, P. W., Tinney, C. G., Burningham, B., et al. 2010, MNRAS, 408, L56

Mainzer, A. K., Eisenhardt, P., Wright, E. L., et al. 2005, Proc. SPIE, 5899, 262

Mamajek, E. E., \& Hillenbrand, L. A. 2008, ApJ, 687, 1264

Mandel, H., Seifert, W., Hofmann, R., et al. 2008, Proc. SPIE, 7014, 70143S

Marsh, K. A., Wright, E. L., Kirkpatrick, J. D., et al. 2013, ApJ, 762, 119

Mason, B. D., McAlister, H. A., Hartkopf, W. I., \& Shara, M. M. 1995, AJ, 109,332

Meixner, M., Smee, S., Doering, R. L., et al. 2010, PASP, 122, 451

Milligan, S., Cranton, B. W., \& Skrutskie, M. F. 1996, Proc. SPIE, 2863, 2
Morley, C. V., Fortney, J. J., Marley, M. S., et al. 2012, ApJ, 756, 172

Pinfield, D. J., Burningham, B., Lodieu, N., et al. 2012, MNRAS, 422, 1922

Rayner, J. T., Cushing, M. C., \& Vacca, W. D. 2009, ApJS, 185, 289

Reach, W. T., Megeath, S. T., Cohen, M., et al. 2005, PASP, 117, 978

Sánchez, S. F., Thiele, U., Aceituno, J., et al. 2008, PASP, 120, 1244

Saumon, D., \& Marley, M. S. 2008, ApJ, 689, 1327

Söderhjelm, S. 1999, A\&A, 341, 121

Stephens, D. C., \& Leggett, S. K. 2004, PASP, 116, 9

Struve, F. G. W. 1827, Catalogus Novus Stellarum Duplicium et Multiplicium (Dorpati [Tartu]: typis J. C. Schuenmanni)

Tinney, C., Faherty, J., Kirkpatrick, J. D., et al. 2012, ApJ, 759, 60

Vacca, W. D., Cushing, M. C., \& Rayner, J. T. 2003, PASP, 115, 389

van Dam, M. A., Bouchez, A. H., Le Mignant, D., et al. 2006, PASP, 118, 310

Wizinowich, P. L., Le Mignant, D., Bouchez, A. H., et al. 2006, PASP, 118, 297

Wright, E. L., Eisenhardt, P. R. M., Mainzer, A. K., et al. 2010, AJ, 140, 1868 